\title{
Approaches for infographics evaluation in maps
}

\author{
Jakub Konicek $^{\mathrm{a}}{ }^{*}$, Vit Vozenilek ${ }^{\mathrm{a}}$, Alena Vondrakova ${ }^{\mathrm{a}}$, Radek Barvir ${ }^{\mathrm{a}}$ \\ ${ }^{a}$ Department of Geoinformatics, Faculty of Science, Palacký University Olomouc, 17. listopadu 50, \\ 77146 Olomouc, Czech Republic; jakub.konicek@upol.cz; vit.vozenilek@upol.cz; alena.vondrakova@upol.cz; \\ radek.barvir@upol.cz. \\ * Corresponding author
}

Keywords: Infographics; Evaluation; Cartography; Maps

\begin{abstract}
:
Cartography is one of the world's oldest sciences. Over time, cartography went through many changes and development phases caused by changing trends and problems, whether social, political, or artistic. Each of the mentioned influences profiled cartography and cartographic creation into the current state. The most visible changes can be recognized in the maps as the main outcomes of cartographic and geographic research. The final artistic style of maps tries to respect the modern graphical trends and ways of data visualization. Nowadays, one of the most recognizable methods is undoubtedly infographics.
\end{abstract}

According to several authors, infographics ideally combine all the advantages of the graphical representation of information. Newson and Haynes (2005) specify infographics as a graphical representation of complex information, data, or knowledge designed to explain them quickly and clearly. This statement is shared in their works by leading experts not only from the world of graphic design and data visualization, especially Mark Smiciklas (2012), Card et al. (1999), Edward Tufte (2007), and Nigel Holmes and Steven Heller (2006), who refer to infographics as "explanatory graphics". In geography and cartography, the term geoinfographics occurs. According to He et al. (2011) or Cheshire and Uberti (2014), it combines thematic map approaches and the general principle of infographics helping users understand works effectively, accurately, and quickly. This complex set of graphic design pieces in one visualization (Krum, 2014) creates universal information design, which can be applied for different kinds of phenomena, including specific spatial data - the same as cartography dealing with.

In the context of cartography, it is not possible to call maps infographics. The map has a clear definition, elements, components, and parts. These can be processed in a certain style. The presented research is dealing with the term infographic style, which can be applied in a certain measure, intensity, and characteristics. The aim is to find out the most proper approach for identifying the most representative elements of the infographic style of the maps, expressing their representation and intensity, and formulate its overall evaluation at the end.

Several approaches using different methodologies were selected for the case study testing their capabilities for infographics evaluation in maps samples. Map samples from various authors, time periods, graphic styles, and methods of cartographic visualization were chosen. These samples enter as a basis for the practical application of chosen approaches.

Qualitative and quantitative evaluation of maps' graphic content characteristics within one global methodological approach is not common. Most authors evaluate specific methods, their variations, design, limitations, or specific parts of the map composition. A limited number of authors deal with a more comprehensive examination of the map from the overall concept, such as evaluation of individual elements used in the map layouts. In the context of identifying infographics in maps as a style applied to its creation, it is necessary to use multifactor metrics that allow a combination of qualitative and quantitative evaluation.

This study verifies approaches that were already used to evaluate maps or graphical content and provides results in both quantitative and qualitative ways. For this purpose, methodology based on Quantitate Content Analysis (QCA) was successfully used in cartography by Muehlenhaus $(2011,2012)$ to evaluate pervasive and propaganda maps. Another approach, visual summary, is a method of visual analysis that enables effective segmentation of sophisticated input data, as multicomponent maps should be. Using affine diagram ideology, the image is segmented into meaningful clusters by applying predefined domains. Manson et al. (2016) used a visual summary approach at uncertainly visualization is a good scope for multicomponent infographics evaluation. Artificial intelligence as part of automatic image processing is a perspective method for evaluation and identification patterns in an image. In cartography, image processing methods are used primarily in remote sensing of the Earth. Few studies also focus on the automatic digitization of colours in thematic maps (Lawrece et al., 1996). 
The motivation of the research is to introduce new custom metrics for infographics evaluation. The proposed methodological approach called 3valuation describes, identifies, and quantifies infographics in maps. It is based on a combination of three components which are evaluated by three criteria within three concepts.

The component is a basic characteristic of the description of the topic. In the 3valuation approach, three components were defined, namely spatial (SP), attribute (AT), and design (DE). Each component consists of a list of compositional elements of the map layout - so-called elements (map field, legend, scale, graph, table, picture, etc.). By changing the list of compositional elements, the 3 valuation approach can be refined in further studies.

The criterion expresses a thematically focused view of the compositional elements of the evaluated infographics. Each appropriately chosen criterion increases the relevance of the evaluation and classification of the infographic. Three criteria were chosen for the 3valuation approach - graphic dominance, area coverage and visual hierarchy. All criteria are based on precisely defined mathematical calculations.

Concepts are the basic elements of a 3valuation approach defining a specific quantification framework for evaluating infographics from the point of view of uniquely constructed combinations of elements. Concepts stand at the top of the 3 valuation approach, consist of components, and differ in their specific composition of compositional elements. Setting up a single concept that would allow universal evaluation of infographics requires a systematic approach to the careful definition of components, criteria, and measurement methods. For applying the 3valuation approach, three unique concepts were compiled and designated. Each of the three compiled concepts was defined by different modifications of the three components, which characterize the features of the infographic design of the map sheets.

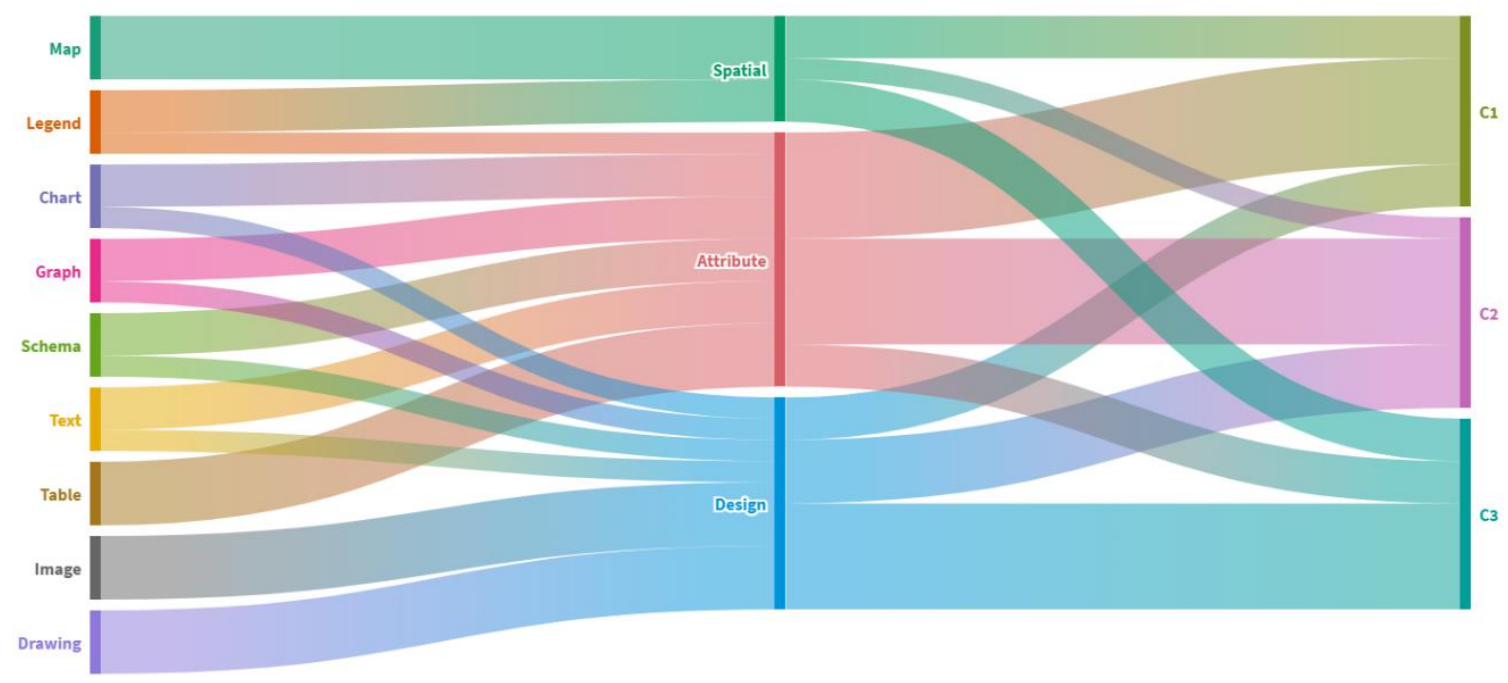

Figure 1: Distribution of elements into the components and concepts

This research aims to verify the usability of individual approaches by realizing the practical case studies, process the acquired knowledge clearly, and provide its comprehensive description for further usage. Such a study aims to deepening knowledge about infographics in cartography and maps itself. Through the proper identification and evaluation methodology, a clarification of this uncertain popular topic could come up. 